\title{
Echocardiography with Elevated Central Venous Pressure Diagnosing Constrictive Pericarditis More Accurately Than Chest CT Scan: A Retrospective Study in 36 Cases
}

\author{
Liwen Bao, MM, Jiechun Huang, MD, Fangrui Wang, MM, Liewen Pang, MM, Yiqing Wang, MD, \\ Haiming Shi, MD \\ Department of Cardiothoracic surgery, Huashan Hospital of Fudan University, 12th Wulumuqi Road, Shanghai, 200040, PR, China
}

\section{ABSTRACT}

Background: Constrictive pericarditis (CP) is defined as impaired diastolic cardiac function caused by a calcified and thickened pericardium. We assessed the clinical characteristics and time to diagnosis, as well as patient prognosis after pericardiectomy.

Methods: We analyzed the records of $36 \mathrm{CP}$ patients who underwent pericardiectomy at Huashan Hospital, China, between 2012 and 2015. Clinical manifestations, length of time to diagnosis, laboratory parameters, and diagnostic imaging results were examined. All patients underwent pericardiectomy, and were assessed post-operatively for quality of life and improvement of cardiac function using the Minnesota Living with Heart Failure Questionnaire (MLHFQ).

Results: All patients displayed shortness of breath and polyserous effusion, as well as elevated pro B-type natriuretic peptide and thickened pericardium. Mean time between onset of symptoms and a definitive diagnosis of CP was $9.5 \pm$ 2.1 months. Pericardiectomy was performed within one week of diagnosis. Mean central venous pressure decreased from a pre-operative $19.92 \pm 6.6 \mathrm{mmHg}$ to a post-operative 8.5 $\pm 2.7 \mathrm{mmHg}$. Within $1.5 \pm 0.7$ years of surgery, all patients maintained good quality of life and cardiac function, which resulted in a mean score of $0.9 \pm 0.6$ on the MLHFQ.

Conclusion: A definitive diagnosis of CP is usually made long after the onset of symptoms. Early detection and diagnosis by echocardiography with elevated central venous pressure and early treatment by surgery are key to an improved prognosis and resumption of good cardiac function.

\section{INTRODUCTION}

Constrictive pericarditis (CP) is defined as heart failure with preserved systolic function; its clinical manifestations include shortness of breath after activity, and enervated, polyserous effusion. CP can be diagnosed from these symptoms,

Received September 11, 2017; received in revised form March 24, 2018; accepted April 20, 2018.

Correspondence: Fiechun Huang, Department of Cardiothoracic surgery, Huashan Hospital of Fudan University, 12th Wulumuqi Road, Shanghai, 200040, PR, China; Tel \& fax: 021-52887066 (e-mail: hjcmdl66@163.com). and imaging of calcification and thickened pericardium. The primary treatment for $\mathrm{CP}$ is pericardiectomy.

$\mathrm{CP}$ is often missed or misdiagnosed because of its insidious onset and infrequency. Although early diagnosis could improve patients' quality of life, many $\mathrm{CP}$ patients are not diagnosed in the early stages of the disease.

We collected data on CP patients to analyze the disease's clinical characteristics, and its diagnosis and treatment methods. We also review the progress made in diagnosing and treating $\mathrm{CP}$, and focus in particular on the role of echocardiography with elevated central venous pressure in assisting clinicians to diagnose $\mathrm{CP}$ early and provide appropriate treatment.

\section{MATERIALS AND METHODS}

This study was conducted at Huashan Hospital (Fudan University, Shanghai, China) as a retrospective study. We analyzed data from $36 \mathrm{CP}$ patients diagnosed from imaging examinations and clinical symptoms, and who underwent pericardiectomy between 2012 and 2015.

We summarized the clinical manifestations, diagnosis methods, examination results, and prognosis in these cases. Clinical manifestations included shortness of breath after activity, palpitations, chest tightness, edema in the lower extremities, pleural effusion, and ascites. Diagnosis methods were based on the results of chest CT scans and detailed findings in echocardiography, electrodiagrams, and laboratory tests (alanine aminotransferase [ALT], aspartate aminotransferase [AST], total bilirubin, conjugated bilirubin, pro B-type natriuretic peptide [proBNP], and central venous pressure before and after pericardiectomy). The length of time to diagnosis was defined as the time that elapsed between the onset of symptoms and confirmation by echocardiography or chest CT scan. The prognosis was assessed from cardiac function and quality of life after surgery through the administration of the Minnesota Living with Heart Failure Questionnaire (MLHFQ) over telephone.

SPSS 17.0 (IBM SPSS, Armonk, NY, USA) was used for statistical analysis. The variables are presented as mean \pm standard deviation for normally distributed data, and as median or percentage for data that were not normally distributed. T-tests and Pearson's chi-squared test were used for group comparisons before and after surgery. $P<.01$ was considered statistically significant. 
Table 1. Clinical features of the 36 patients

\begin{tabular}{lc}
\hline Variables & Findings \\
\hline Baseline & \\
Male Sex & $34(94.2 \%)$ \\
Age (years) & $40.2 \pm 5.6$ \\
$\quad$ Time to diagnosis (months) & $9.5 \pm 2.1$ \\
Clinical Manifestations & \\
Shortness of breath & $34(94.4 \%)$ \\
Fatigue & $18(50 \%)$ \\
Palpitation & $4(11.1 \%)$ \\
Edema in lower extremities & $36(100 \%)$ \\
Ascites & $20(55.6 \%)$ \\
Pleural effusion & $18(50 \%)$ \\
Laboratory Findings & \\
Atrial fibrillation & $4(11.1 \%)$ \\
Alanine aminotransferase $(\mathrm{U} / \mathrm{L})$ & $22.06 \pm 3.55$ \\
Aspartate aminotransferase $(\mathrm{U} / \mathrm{L})$ & $22.75 \pm 3.26$ \\
Total bilirubin $(\mu \mathrm{mol} / \mathrm{L})$ & $29.33 \pm 8.48$ \\
Conjugated bilirubin $(\mu \mathrm{mol} / \mathrm{L})$ & \\
Troponin $(\mathrm{c}-\mathrm{TNT}) \mathrm{ng} / \mathrm{ml}$ & $0.064 \pm 0.02$ \\
Pro B-type natriuretic peptide $(\mathrm{pg} / \mathrm{mL})$ & $622.78 \pm 135.74$ \\
& \\
\hline &
\end{tabular}

RESULTS

We analyzed data from 36 patients. Mean patient age was $40.2 \pm 5.6$ years; 34 patients were male $(94.4 \%)$. All patients had been diagnosed with CP and underwent pericardiectomy between 2012 and 2015 in Huashan Hospital, Fudan University. Table 1 displays the clinical features of the patients.

Almost every patient complained of shortness of breath, palpitations, and fatigue. Edema in both lower extremities was found in all patients, and polyserous effusions of various degrees were confirmed via imaging. Ultrasonography of patients with abdominal distension revealed moderate to large ascites. Chest CT scans showed moderate to large pleural effusion and mild pericardial effusion in 13 patients (36.1\%) who complained of severe chest congestion after activity; $1,400 \mathrm{~mL}$ of pleural effusion was removed from one patient during surgery, and ascites combined with pleural effusion were found in three others. Apart from moderate to large polyserous effusion, two other cases showed mild polyserous effusion in imaging examinations; one 31-year-old patient complained of having slight chest congestion with edema in the lower extremities for 24 months, and one 46-year-old patient complained of having chest congestion after activity for 4 months.

Electrocardiograms found T-wave reversion in leads II, III, avF, and V4-6, indicating abnormal ventricular repolarization at the inferior and lateral wall of the left ventricle in each patient. Paroxysmal atrial fibrillation was found in
Table 2. Imaging Findings in 36 Patients

\begin{tabular}{lc}
\hline Findings & $\begin{array}{c}\text { Number of patients } \\
\text { or value found }\end{array}$ \\
\hline Echocardiography & \\
Bilateral Atrial Enlargement & $31(86.1 \%)$ \\
Inferior Vena Cava Broadening & $23.5+2.1 \mathrm{~mm}$ \\
Pericardial Thickness & $36(100 \%)$ \\
Diastolic Function Restriction & $36(100 \%)$ \\
Ventricular Septal Jitter & $22(61.1 \%)$ \\
Mitral Valve Flow Pattern Changes With Respiration & $31(86.1 \%)$ \\
Mitral Valve Flow Pattern Normalized & $29(80.6 \%)$ \\
Mitral Valve Flow Restriction & $2(5.6 \%)$ \\
Chest CT & \\
Pericardial Thickening & $27(75 \%)$ \\
Pericardial Calcification & $9(25 \%)$ \\
Pleural Effusion & $18(50 \%)$
\end{tabular}

four patients whose diagnosis period exceeded 12 months. Some common abnormalities were found in the laboratory tests, such as elevated troponin (c-TNT) and proBNP in 36 patients, and increased total bilirubin and conjugated bilirubin with normal ALT and AST in 29 patients. Other laboratory examinations, including a complete blood count, renal function, and electrolyte levels, were in the normal range.

All 36 patients underwent echocardiography and chest CT scans: 29 patients were diagnosed from echocardiography, and seven patients were diagnosed from the pericardial thickening found in CT scans and confirmed by echocardiograms (Table 2). Chest CT scans revealed partial pericardial thickening of the posterior and lateral wall of the left ventricle in 27 patients, nine of whom exhibited pericardial calcification. No pericardial thickening was observed in the chest CT scans of nine patients. Echocardiography showed diastolic function restriction and increased echogenicity in the posterior and lateral wall of the left ventricle in all 36 patients, as well as an enlarged inferior vena cava with little change during respiration. Ventricular septal jitter with respiration was observed in in 22 patients, and some patients displayed pseudo-normalized or restrictive physiologic mitral valve flow changes with respiration.

The shortest period to diagnosis in the cohort was 2.5 months, and the longest period exceeded 24 months (seven patients); the mean period was $9.5 \pm 2.1$ months. Shortness of breath after activity was often misdiagnosed initially as dilated cardiomyopathy or pneumonia.

During the period from definite diagnosis to surgery, all of the patients were treated with thiazine diuretic. The dose of thiazine diuretic ranged from $5 \mathrm{mg}$ qd to $20 \mathrm{mg}$ bid through intravenous or oral administration. The features of edema were relieved to some extent, while other symptoms like shortness of breath, palpitations, and fatigue continued without improvement. Two patients were treated with digoxin.

Pericardiectomy was performed within one week of 
Table 3. Cardiac Function Pre- and Post-Pericardiectomy

\begin{tabular}{lccc}
\hline Variables & Pre-surgery & Post-surgery & $P$ \\
\hline Central Venous Pressure $\left(\mathrm{cmH}_{2} \mathrm{O}\right)$ & $19.92 \pm 6.6$ & $8.5 \pm 12.7$ & $<.01$ \\
Left Ventricular Ejection Fraction $(\%)$ & $56 \%$ & $58 \%$ & .86 \\
Systolic Pressure $(\mathrm{mmHg})$ & 130.2 & 127.6 & .85 \\
Cardiac Index $\left(\mathrm{L} / \mathrm{min} / \mathrm{m}^{2}\right)$ & 2.9 & 3.1 & .91
\end{tabular}

diagnosis. Pericardial thickening of $1-2 \mathrm{~cm}$ was found mostly at the posterior and lateral walls of the left ventricle in all patients, and pericardial calcium was observed in 11 patients during pericardiectomy. Mean central venous pressure was elevated before the procedure $\left(19.92 \pm 6.6 \mathrm{cmH}_{2} \mathrm{O}\right)$, and decreased to $8.5 \pm 2.7 \mathrm{cmH}_{2} \mathrm{O}$ after the procedure (Table $3)$. Patients were discharged within one week of pericardiectomy without complications such as pneumonia or lower cardiac output. Consistent with clinical diagnosis, the pathology showed tuberculosis in seven cases, including pulmonary tuberculosis in four patients and tuberculous peritonitis in one patient. The pathology in the other 29 patients showed only hyperplasia of collagen fibers, hyaline and chronic inflammation, or lymphocytic infiltration.

Cardiac function recovered soon after surgery; no patients died or were re-hospitalized during a follow-up period of 4.8 \pm 1.2 years. All 36 patients reported good quality of life on the Minnesota questionnaire, which resulted in a mean score of 0.9 \pm 0.6 on the MLHFQ. Normal left ventricular structure and function were confirmed by echocardiography in 29 patients.

\section{DISCUSSION}

$\mathrm{CP}$ is characterized as a ventricular filling disorder, causing an increase in venous pressure and Kussmaul breathing, derived from pericardium adhesion to the parietal layer[Clare 2007]. The etiology of CP includes tuberculous pericarditis, viral pericarditis, pericardial injury after surgery, radiation, cytoskeletal disease, and idiopathic pericarditis[Chang 2017; Jiang 2013; Szabó 2013]. Tuberculous pericarditis is more prevalent in developing nations, while idiopathic pericarditis and pericardial injury after surgery have mainly been reported in developed nations.

Previous studies have indicated that young adults are prone to CP, and the prevalence is higher in [Schwefer 2009]. Cardiac diastolic dysfunction and increased venous pressure lead to symptoms such as jugular venous distension, pleural effusion, ascites, hepatomegaly, shortness of breath after activity, and fatigue [Welch 2017].

Laboratory findings revealed elevated bilirubin levels resulting from congestion in bile canaliculi because of increased venous pressure. Elevated c-TNT and proBNP indicates impairment in the myocardium from partial pericarditis, showing the degree of systemic circulation. Clinicians should pay attention to these laboratory findings if combined with diastolic cardiac dysfunction, as this could assist in making a correct diagnosis. The period from onset of symptoms to diagnosis averaged 9 months in our cohort; this is longer than the times for other diseases because of CP's atypical symptoms and lack of recognition by clinicians. Echocardiography and chest CT scans were used to diagnose $\mathrm{CP}$ definitively, though echocardiography was proved to be more accurate than chest CT scan.

Nonspecific ST-T wave changes imply myocardial impairment. In our cohort, ventricular repolarization of the inferior and lateral wall indicated in electrocardiograms corresponded to the findings in pericardiectomy, which showed adhesion and calcification, mostly on the posterior wall. Chest CT scans, cardiac MRI, and echocardiography are the main imaging techniques used to diagnose $\mathrm{CP}$ [Klein 2013]. Chest CT scans can detect pericardial thickening $(>4$ $\mathrm{mm}$ ) and even minute calcification, while other findings may include a normal or slightly reduced ventricular chamber, enlarged inferior vena cava, hepatic vein, and right atrium, hepatosplenomegaly, ascites, and pleural effusion. Cardiac MRI can detect pericardial thickening, ventricular diastolic restriction, ventricular septal jitter, pericardial edema, and pericardial inflammation; however, cardiac MRI is less effective at detecting calcification. Chest CT scans in our cohort detected thickened pericardium in $75 \%$ of cases, pericardial calcification in $25 \%$ of cases, and no abnormal findings in the other $25 \%$ of cases, which may be attributed to physicians' unfamiliarity with pericardial disease. This lack of awareness can delay early diagnosis and treatment of the disease.

Echocardiography is the most important noninvasive diagnostic method for CP. Two-dimensional and M-mode echocardiography can detect pericardial thickening and calcification, atrial enlargement, a broadened inferior vena cava, ventricular septal jitter, and normal left ventricular systolic function [Klein 2013]. Mitral rheography can show a significantly increased E peak, decreased A peak, shortened deceleration time, and decreases of more than $25 \%$ during inspiration [Liu 2014; Liu 2013]. Tricuspid valve blood flow can indicate decreased expiratory flow of more than 40\%-60\%.

The pulmonary venous velocities' changes are similar to those of the mitral valve. The hepatic venous velocity shows increased diastolic flow during expiration, reflecting the separation between intracardiac pressure and intrathoracic pressure. The clinical features of $\mathrm{CP}$ are similar to those of restrictive cardiomyopathy, but tissue Doppler imaging can be used to differentiate between the two diseases [Mookdam 2011]. Rheography of restrictive cardiomyopathy yields a thin, high E wave that appears shorter than $7 \mathrm{~cm} / \mathrm{s}$ in Doppler imaging, and hepatic venous velocity shows increased diastolic flow during inspiration. In contrast, Doppler changes in CP include a low E/e' ratio, a reversed left atrial pressure curve, a septal $\mathrm{e}^{\prime}$ wave which gradually increases with advance of pericardial constriction, and a lower $\mathrm{e}^{\prime}$ wave in the posterior wall than in the septal wall. To avoid misdiagnosis, chest CT scans and cardiac MRI can be used to differentiate between the two diseases, if echocardiography is insufficient [Xiong 2013].

Pericardiectomy is the only definitive treatment for $\mathrm{CP}$ [Lee 2018; Chen 2014]. Our cohort underwent surgery by median sternotomy incision. Because of adequate preoperative evaluation, no organs were impaired. Proper surgical 
protocol, appropriate post-operative nursing, minimal diuretics, and adequate nutritional and liquid support prevented complications of pleural effusion, pulmonary infection, and low cardiac output in the 36 patients, who were discharged within one week of the surgery [Motoki 2013].

The pathology showed tuberculous pericarditis in seven patients, and idiopathic pericarditis in the other 29 cases, which is consistent with data from previous reports [Szabó 2013]. The patients were all residents of Shanghai and the Yangtze River Delta, an area of high economic development, and exhibited positive lifestyle habits and health awareness. Thus, the main etiology of CP was not tuberculosis, but some idiopathic cause.

The patients were followed for $4.8 \pm 1.2$ years. All 36 were able to care for themselves, and participated in normal physical exercise, which resulted in a mean score of $0.9 \pm$ 0.6 in MLHFQ years. 29 received follow-up echocardiography, which indicated normal cardiac structure and function. Through immediate and proper treatment, CP patients have a good prognosis [Syed 2014]. Other retrospective analyses of hospital records found similar results [Avgerinos 2014; Lin 2012]. Good prognosis for CP cases involves accurate diagnosis and proper treatment.

Our study is limited in that it was a single-center retrospective study with a relatively small sample size, and therefore cannot fully reflect the current conditions of the disease in the region.

\section{CONCLUSION}

Early diagnosis by echocardiography with elevated central venous pressure, and treatment by timely pericardiectomy, are key to a good prognosis in CP. When patient symptoms are caused by suspected diastolic heart failure, clinicians must make an imaging examination, especially echocardiography, as soon as possible, so as to make a differential diagnosis. Once the diagnosis made, pericardiectomy should be performed in a timely manner to attain a good prognosis in the future.

\section{REFERENCES}

Avgerinos D, Rabitnokov Y, Worku B, et al. 2014. Fifteen-year experience and outcomes of pericardiectomy for constrictive pericarditis. J Card Surg 29:434-8.

Chang SA. 2017. Tuberculous and Infectious Pericarditis. Cardiol Clin 35:615-622.
Chen H. 2014. Analysis of effects of different approaches of pericardiectomy for constrictive pericarditis. China Modern Medicine 21: 29-30.

Clare GC, Troughton RW. 2007. Management of constrictive pericarditis in the 21st century. Curr Treat Options Cardiovasc Med 9:436-442.

Jiang Y, Shao F. 2013. A stone miner with both silicosis and constrictive pericarditis: case report and review of the literature. BMC Pulm Med 13:71.

Klein AL, Abbara S, Agler DA, et al. 2013. American Society of Echocardiography clinical recommendations for multimodality cardiovascular imaging of patients with pericardial disease: endorsed by the Society for Cardiovascular Magnetic Resonance and Society of Cardiovascular Computed Tomography. J Am Soc Echocardiogr 26:9651012. e15.

Lee Y, Naruse Y, Tanaka K. 2018. Effectiveness and long-term outcomes of surgical intervention for constrictive epicardium in constrictive pericarditis. Gen Thorac Cardiovasc Surg. 66:137-44.

Lin Y, Zhou M, Xiao J, et al. 2012. Treating constrictive pericarditis in a Chinese single-center study: a five-year experience. Ann Thorac Surg 94:1235-40.

Liu K, Deng YB, Sun J, et al. 2014. Evaluation of cardiac function in patients with constrictive pericarditis by two-dimensional speckle tracking echocardiography. Acta Med Univ Sci Technol Huazhong 43: 90-93.

Liu K, Deng YB. 2013. Progresses of ultrasonic diagnosis of constrictive pericarditis. Chin J Interv Ther 10:116-119.

Mookadam F, Jiamsripong P, Raslan SF, et al. 2011. Constrictive pericarditis and restrictive cardiomyopathy in the modern era. Future Cardiol 7:471-83.

Motoki H, Alraies MC, Dahiya A, et al. 2013. Changes in left atrial mechanics following pericardiectomy for pericardial constriction. J Am Soc Echocardiogr 26:640-8.

Schwefer M, Aschenbach R, Heidemann J, et al. 2009. Constrictive pericarditis, still a diagnostic challenge: comprehensive review of clinical management. Eur J Cardiothorac Surg 36:502-10.

Syed FF, Schaff HV, Oh JK. 2014. Constrictive pericarditis--a curable diastolic heart failure. Nat Rev Cardiol 11:530-44.

Szabó G, Schmack B, Bulut C, et al. 2013. Constrictive pericarditis: risks, aetiologies and outcomes after total pericardiectomy: 24 years of experience. Eur J Cardiothorac Surg 44:1023-8.

Welch TD. 2017. Constrictive pericarditis: diagnosis, management and clinical outcomes. Heart 0:1-7.

Xiong QF, Ma XJ, Chen Y. 2013. Clinical value of MSCT in the diagnosis of constrictive pericarditis. Radiologic Practice 4:022. 